\title{
Report on the fourth international workshop on the CCN family of genes
}

\author{
S. Kubota $\cdot$ H. Yeger $\cdot$ B. Perbal $\cdot$ M. Takigawa
}

Received: 1 February 2007 / Accepted: 2 February 2007 / Published online: 6 April 2007

(C) The International CCN Society 2007

Keywords CCN $\cdot$ Genes $\cdot$ Proteins

The Fourth International Workshop on the CCN Family of Genes, organized by Prof. Masaharu Takigawa and Prof. Bernard Perbal was held October 18-22, 2006 in Ushimado, Setouchi-city, Okayama, Japan. The meeting venue at the Hotel Limani in Ushimado provided elegant surroundings and an atmosphere that encouraged close interaction and discussion among the participants as well as a unique environment to enjoy the finest in Japanese hospitality.

Opening remarks from Prof. Perbal and Prof. Takigawa nicely set the stage for the ensuing platform presentations and poster session where numerous aspects of $\mathrm{CCN}$ biology, disease relevance, and opportunities for therapeutics were covered.

In Session I, on Regulation of Gene Expression, B. Chaqour (SUNY, Downstate Medical Center, NY) reported on rapid induction of $\mathrm{CCN} 2$ expression in cyclically

S. Kubota $\cdot$ M. Takigawa

Department of Biochemistry and Molecular Dentistry, Graduate

School of Medicine, Dentistry and Pharmaceutical Sciences,

Okayama University,

Okayama, Japan

H. Yeger

Department of Paediatric Laboratory Medicine,

The Hospital for Sick Children,

Toronto, Canada

B. Perbal $(\square)$

Laboratoire d'Oncologie Virale et Moléculaire,

Université Paris 7 - D. Diderot,

Paris, France

e-mail: perbal@ccr.jussieu.fr stretched bladder smooth muscle cells and involvement of $\mathrm{NF}-\mathrm{KB}$ therein. CCN2 induction was highly dependent on integrity of the actin network and cytoskeletal organization reinforcing the idea that mechanical movements modulate $\mathrm{CCN} 2$ expression. Further studies were presented on an in vivo model of bladder outlet obstruction where upregulation of CCN2 may account for pathological alterations after mechanical overload. S Kubota from Prof. Takigawa's group (Dept. of Biochemistry and Molecular Dentistry, Okayama University) expanded upon previous studies showing that the 3 -untranslated regions (UTRs) of CCN genes contain post-transcriptional repressor elements that regulate the intracellular stability of $\mathrm{CCN}$ mRNAs, and in CCN4 there also is a specific regulator of gene expression operative in both chondrocytic and osteoblastic cells. This helps to explain the tissue/stage-specific production of $\mathrm{CCN}$ proteins from the cells where their activity may be critical for differentiation.

H. Yeger (Hospital for Sick Children, Toronto) presented recent studies on $\mathrm{CCN} 3$ expression in teratocarcinoma NT2D1 cell line model grown on an ECM like polyamide substrate (UltraWeb) as analyzed by immunofluorescence labeling and confocal microscopy. CCN3 expression was correlated with other markers of neurogenesis during retinoic acid induction. Emphasis was placed on the importance of utilizing more 3D like in vitro models to study CCN expression, since these systems more closely mimic tissue structures. Observations suggested that CCN3 was upregulated in cell types, possibly glial, accompanying terminally differentiated neurons.

In Session II on Receptors and Signaling Pathways, T. Nishida (Dept of Biochemistry and Molecular Dentistry, Okayama University) spoke about how CCN2 mediates fibronectin binding to $\alpha 5 \beta 1$ integrin and sustains integrin 
signaling. The question was raised whether fibronectin was directly involved in modulating CCN2 activity, since endogenous fibronectin expression was not different between wild type and CCN2 knockout cells.

T. Hattori (Dept of Biochemistry and Molecular Dentistry, Okayama University) expanded further on the association of CCN2 with fibronectin and presented new data on potential new binding partners for $\mathrm{CCN} 2$ in human chondrocytes. CCN2 enhanced adhesion of HCS-2/8 to fibronectin via $\alpha 5 \beta 1$ integrin. A new binding partner, Matrilin 3, a non-collagenous ECM protein, was shown to be involved, but its actual role is still unclear. However, $\mathrm{CCN} 2$ can be both growth factor and stabilizer of ECM structure and facilitates binding of cells with CCN2 activities mediated through its CT domain.

K-I Katsube, (Tokyo Medical and Dental University), provided further insight into the role of Notch signaling and its facilitation by $\mathrm{CCN} 3$ in the growth and differentiation of bone marrow derived mesenchymal stem cells (Kusa A-1 cell line). In lung these cells undergo osteogenesis while the KusaA(NICD) subline does not form osteogenic nodules but is directed towards neurogenesis with increase in Hes-1. Notch suppressed cell line, Kusa CBF1/Kusa-A1, showed decreased $\mathrm{CCN} 3$. Microarray analysis revealed that $\mathrm{CCN} 3$ expression increased with upregulation of Notch. CT truncated $\mathrm{CCN} 3$ favored osteogenesis, while N-terminal truncated $\mathrm{CCN} 3$ favored neurogenesis coordinating with upregulation of other neural transcription factors, Hey-1 and Dec. Thus Notch appears to coordinate tissue type specific differentiation activities via $\mathrm{CCN} 3$, and the various domains of $\mathrm{CCN} 3$ may affect developmental decisions. This domain specification theme is elaborated upon in the continuing sessions.

In the first of two Sessions on Cell Growth and Differentiation, B. Perbal (Laboratoire d'Oncologie Virale et Moleculaire, Universite Paris 7) highlighted provocative new data supporting the role of $\mathrm{CCN} 3$ as an antiproliferative $\mathrm{CCN}$ family member. Co-culture of melanocytes and keratinocytes (simulating the skin arrangement) induced CCN3 secretion. Whereas IL- $1 \beta$ and TNF- $\alpha$ induced $\mathrm{CCN} 3$, infection of melanocytes with adeno- $\mathrm{CCN} 3$ suppressed growth as did exogenous CCN3. It would appear that keratinocytes inhibit the migration and invasion of melanocytes via actions of CCN3. In other studies the CT domain was shown to be strongly growth inhibitory, and since it contains a cystine knot equivalent to other growth factors like NGF, IGFBP2, PDGF $\beta$ and HCG, it begs the question whether $\mathrm{CT}$ domain of $\mathrm{CCN} 3$ can dimerize with other growth factors and thus modulate their activity, or not. Along the same line, B. Perbal proposed that heterodimerization of $\mathrm{CCN}$ proteins may be governed at least in part by the CT domain, and that this plays a crucial role in regulating the biological activities of $\mathrm{CCN}$ proteins. The question then arises whether small differences in CT sequence as found in the different $\mathrm{CCN}$ proteins determines binding partners and ultimate functional outcomes which can be quite dissimilar and perhaps competitive at the ECM level. Other data was presented showing that a time dependent increase in intracellular CCN3 correlated with cell density and cessation of growth. CCN3 protein that is very stable and accumulates continuously was found to be increased during $\mathrm{G} 2 / \mathrm{M}$ transition while expression levels were regulated by recycling activity in the cell. C. Naus, (Dept of Cellular and Physiological Sciences, University of British Columbia), updated everyone on the striking effects of CCN1 on C6 glioma cells and interaction with gap junction protein $\mathrm{Cx} 43$. Serum stimulation of $\mathrm{Cx} 43$ transfected $\mathrm{C} 6$ cells upregulated and prolonged $\mathrm{CCN} 1$ expression. However, CCN1 was diffusely distributed in cells and not associated with $\mathrm{Cx} 43$ at gap junctions. Overexpression of CCN1 increased growth rate of C6 cells. Since CCN3 has been previously associated with $\mathrm{Cx} 43$, with binding to the C-terminal end of $\mathrm{Cx} 43$, and is associated with growth inhibition, CCN1 might operate through a metabolic mechanism. Does this evidence suggest that, at least in cancer cells, expression of multiple CCN proteins would be counterproductive for continuous cancer cell growth and tumorigenic behavior?

In the second Session on Cell Growth and Differentiation, W.C. Sin, (University of British Columbia), from Dr. Naus's laboratory presented new information on the interaction of $\mathrm{CCN} 3$ with $\mathrm{Cx} 43$, this time in breast cancer cell lines, MDA-MB-231 and Hs578T. Full length CCN3 is expressed and secreted, less in the more aggressive MB231 and more in the less aggressive Hs578T cell lines. The phosphorylation rich C-terminal region of $\mathrm{Cx} 43$ is involved in binding with $\mathrm{CCN} 3$, and when truncated, very little CCN3 is co-immunoprecipitated. Cx43 siRNA treatment of Hs578T downregulated CCN3 cytoplasmic and secreted productions. Interestingly, transfection with $\mathrm{C}$-term truncated $\mathrm{Cx} 43$ forced nuclear localization of $\mathrm{CCN} 3$ suggesting that $\mathrm{CCN} 3$, as recently reported, may be transcriptionally active in some as yet undefined manner. In some ways this mimics what occurs with $\beta$-catenin. In other experiments, transfection of CCN3 NH24 (domains 1-3 inclusive) into MB-231 increased growth and migration suggesting that the N-terminal end of $\mathrm{CCN} 3$ imparts different properties to CCN3 in an obvious cell context manner. S. Twigg, (Discipline of Medicine, University of Sydney, Australia), elaborated, with extensive data, on the role of CCN2 in adipogenesis and adipocyte differentiation. The 3T3-L1 cell line (adipogenic cell line model) shows downregulation of $\mathrm{CCN} 2$ upon induced adipocyte differentiation. Treatment with $\mathrm{CCN} 2$ reversed the differentiation where $\mathrm{C} / \mathrm{EBP}-\mathrm{b}$ transcription factor was downregulated. TGF- $\beta 1$ had a similar effect in reducing expression of adipocyte relevant 
PPAR and adiponectin. In fat fed mice, CCN2 is increased in central adipose tissue but not in cutaneous fat. Thus CCN2 may play an important role in inhibiting fat cell differentiation while promoting increase in ECM and fibrous tissue. Similarities and differences that exist amongst the different arms of the mesenchymal lineage where $\mathrm{CCN} 2$ and other $\mathrm{CCN}$ proteins can play prominent roles in cell fate determination need to be explored in greater depth since this may have great clinical import. The Session ended with a presentation from J. Malmquist, (Dept Anatomy and Cell Biology, Tufts University School of Medicine, Boston), speaking on the role of CCN5 as a suppressor of restenosis in a mouse model of vascular injury where hyperproliferation of smooth muscle cells (SMC) is a pathological feature. CCN5 overexpression resulted in the reduction of proliferation, motility and MMP2 gene expression in those cells. In normal SMC, $\mathrm{CCN} 3$ is upregulated while it is downregulated during injury. CCN5 is expressed in SMC that are growth arrested and also in endothelium. Thus CCN3 and CCN5 appear to mimic each other in their antiproliferative abilities. In a carotid ligation model (restenosis) CCN5 increased in the neointima and decreased in the media. Overexpression of CCN5 reduced and eliminated the neointima but the media was not affected. So, CCN5 appears to be an important regulator in this pathological process. Some variable staining with anti-CCN antibodies in this study continues to address antibody specificity and thus the need for standardized reagents for the CCN field.

In the first Session on Development, Remodeling and Regeneration, M. Takigawa, (Dept of Biochemistry and Molecular Dentistry, Okayama University), presented a broader overview of the role of $\mathrm{CCN}$ family proteins in skeletal growth and regeneration. This presentation provided a better perspective on an area that has been pioneered by Takigawa's group in the demonstration that multiple CCN 2,3,4 and 6 proteins contribute to chondrogenesis and osteogenesis with transduction of growth and differentiation signals mediated by MAPK and PI3K pathways. A variant of CCN4 with VWC domain spliced out enhanced the hypertrophic phenotype begging the question whether this type of variant exists for other $\mathrm{CCN}$ types, or not. Overall there also appears to be extensive recycling and functional inactivation of $\mathrm{CCN}$ protein upon terminal differentiation suggesting that $\mathrm{CCN}$ proteins are principally needed during high metabolic activity and cellular remodeling. Clinically relevant, $\mathrm{CCN} 2$ was shown to stimulate expression and production of elastin, which is a marker of elastic cartilage, in cultured auricular chondrocytes. Moreover, when chondrocytes pelleted in culture were immersed with $\mathrm{CCN} 2$ before implantation into mouse back skin, cartilage formation was markedly promoted. Thus there is promise of significant clinical applications stemming from
CCN work. K. Lyons, (Dept of Orthopaedic Surgery, UCLA), followed by an in depth molecular analysis exploiting null or floxed ccn1 and ccn2 mouse models to understand the biological functions of $\mathrm{CCN} 1$ and $\mathrm{CCN} 2$ in skeletal growth and vascularization. $\mathrm{CCN} 1$ was found to be required for radial-humoral joint formation and $\mathrm{CCN} 2$ for formation of the growth plate. CCN1 was expressed in articular cartilage and $\mathrm{CCN} 2$ in the subarticular region. Interestingly, $\mathrm{CCN} 1+/-/ \mathrm{CCN} 2-/-$ crossed mice showed bigger differences in ossification of vertebrae than CCN2-/- mice alone suggesting co-operation amongst $\mathrm{CCN}$ proteins and possible dose dependent effects. Data was also presented on the effects of $\mathrm{CCN} 2$ deletion on angiogenesis of skin, (CCN2 is highly expressed by epidermal stem cells); here blood vessels were structurally affected and dermal fibroblasts showed poor contractility in an in vitro collagen assay. Further analysis showed that $\mathrm{CCN} 2$ is needed for production of basement membrane type IV collagen signaled through PI3K/Akt. These studies provide further dramatic evidence that $\mathrm{CCN}$ proteins, although being closely structurally related, have distinct functions and are critical for production and structural organization of ECM. A. Kudo, (Dept of Biological information, Tokyo Institute of Technology), ended this Session with further insights into the behavior of CCN proteins under mechanical stress, in this case, the action of CCN3 cooperating with periostin, on periodontal ligament and periosteum. $\mathrm{CCN} 3$ binds to the FAS1 domain of periostin and together they complex with Notch1. Thus in periostin $-/-$ mice Notch 1 and $\mathrm{CCN} 3$ are downregulated in periodontal ligament. Furthermore, CCN3 binds to Notch1 precursor protein as does periostin. Thus, this study introduces another interactive player in the mechanism of CCN3 function.

In the second Session on Development, Remodeling, and Regeneration, the focus was essentially on the role of CCN2 in bone formation. T. Kuboki, (Dept of Oral and Maxillofacial Rehabilitation, Okayama University), addressed the clinical potential for CCN2 in alveolar bone regeneration. CCN2 treated human bone marrow stromal cells cultured on porous hydroxyapatite carriers showed enhanced attachment onto and migration into the matrix as well as increased survival. Such effects of $\mathrm{CCN} 2$ could be mediated by the $\mathrm{CT}$ module alone. $\mathrm{CCN} 2$ therefore shows promise for clinical application for dental prosthetics through the regeneration of alveolar bone. M. Kanyama, (Orthopaedic Surgery, Thomas Jefferson University Medical School, Philadelphia), took a wider look at the role of CCN2 in odontogenesis in the mouse model. CCN2 mRNA was localized by ISH to epithelial and mesenchymal cells during multiple stages of odontogenesis but was downregulated in enamel secreting ameloblasts, similar to BMP-4 expression patterns. Using BMP-4 coated beads, 
CCN2 expression was found to be induced in both dental and non-dental mesenchyme at comparative 10 fold lower concentrations respectively. CCN2 stimulated proliferation of mandibular mesenchymal cells in a dose dependent manner. Thus CCN2 drives growth of odontogenic mesenchyme as induced by BMP-4, via MSX-1 (blocked by Noggin) and then is turned off in mature tooth. Again, this appears to be a recurring theme for $\mathrm{CCN}$ proteins where they are essential or even obligatory for the formative stages in tissue development but where local concentrations may dictate different biological outcomes. It should be also noted that, not only CCN2, but also CCN1, 3, 4 and 5 were shown to be involved in tooth development process. T. Takano-Yamamoto, (Div of Orthodontics and Dentofacial Orthopaedics, Tohoku University), using a mouse model showed that CCN2 expression correlated with induction of apoptosis in alveolar osteocytes that were subjected to mechanical stress. On the pressure side of alveolar bone transient peak in CCN2 and gradual reduction in CCN2 level was correlated with increase in apoptotic osteocytes. The tension side did not show any of this. Osteoclasts numbers also increased on the pressure side indicating active remodeling. Thus $\mathrm{CCN} 2$ expression reacts transiently to tooth movement but it is still unclear whether this is purely reactive or has functional significance.

Session VII, first Session on Tumor Biology, then shifted gears with the presentation by R. Lupu, (Robert H Lurie Comprehensive Cancer Center, Northwestern University, Evanston, IL), who addressed the clinically observed progression of breast cancer from estrogen dependent, (responds to hormone therapy), to independent states, (estrogen resistant). The well differentiated MCF-7 breast cancer cell line, (estrogen receptor positive), transfected with $\mathrm{CCN} 1$ induced estrogen resistance and cells became tumorigenic and metastatic; this now matched the behavior of MDA-MB-231 which has high CCN1 expression. It was further shown that $\mathrm{CCN} 1$ binding sites to $\alpha \mathrm{V} \beta 3$ and $\alpha 6 \beta 1$ integrins were essential for this phenotypic change. In clinical samples $\mathrm{CCN} 1$ is upregulated in node positive breast cancers but does not correlate with Her-2 or PgP. Targeting of tumor cells lines with Taxol plus an RGD-peptidomimetic agent, (anti-integrin, S247, Pfizer) synergistically abrogated the tumorigenic and metastatic patterns of MDA-231 in a xenograft model. Finally, Novus antibodies to CCN1 domains showed diverse patterns in immunoprecipitates from nuclei suggesting that $\mathrm{CCN} 1$ likely resides in different complexes with different partners in the nucleus. This further suggests possible diverse transcriptional targets and raises similar possibilities for other CCN proteins. The subsequent two speakers, S. Irvine and L. McCallum, (both Queen's University, Dept of Haematology Research, Belfast), presented new insights into the role of CCN3 in chronic myeloid leukaemia (CML). S. Irvine has previously reported downregulation of $\mathrm{CCN} 3$ as a consequence of BCR-ABL kinase activity, (the pathognomonic marker of CML). Transfection of CCN3 into K562 cells significantly reduced clonogenicity in methyl cellulose and pushed cells into apoptosis. This was mimicked by application of exogenous $\mathrm{CCN} 3$ protein to $\mathrm{K} 562$ cells. Furthermore, $\mathrm{CCN} 3$ expression increases in patients treated with Imatinib with an initial lower molecular weight $\mathrm{CCN} 3$ band detected, which disappears with treatment. L. McCallum studied this relationship further and showed that BCR-ABL siRNA treatment of K562 decreased $\mathrm{BCR}-\mathrm{ABL}$ and upregulated $\mathrm{CCN} 3$ expression, which was mimicked by Imatinib treatment. Patients are negative for full length CCN3 at diagnosis but a high molecular weight form is upregulated. Patients that do not respond to Imatinib resemble the BCR-ABL state in terms of CCN3. Patients with high CCN3 at diagnosis did not achieve molecular remission. Another line, HL60 also showed CCN3 band differences upon differentiation. Since CCN3 level is relatively high (shows a wide range) in normal marrow and blood, a relevant question here is whether CML patients are generally deficient in circulating $\mathrm{CCN} 3$, since endothelium can secrete $\mathrm{CCN} 3$. Is there resistance to $\mathrm{CCN} 3$ and could $\mathrm{CCN} 3$ be administered therapeutically at least for some patients? Could other $\mathrm{CCN}$ types that are also anti-mitogenic override resistance to CCN3 in selected patients? A propos the above question, S. Banerjee, (University of Kansas Medical Center), closed the Session with another provocative presentation of CCN5 and its role in the progression of breast cancer where mutant p53 predominates. Interestingly, acquisition of mutant p53 during breast cancer progression may impart CCN5 with tumor suppressor activity. Mutant p53 transfection of MCF-7 cells, which are E-cadherin rich, drives down CCN5 expression as well as E-cadherin, shifting the phenotype to that resembling MDA-231. CCN5 siRNA treatment also mimicked the situation of acquired aggressive phenotypes. So silencing of CCN5 expression may be associated with acquisition of the metastatic phenotype. Taken together, data from this Session places greater importance on determining how small changes in $\mathrm{CCN}$ sequence, as found in the different $\mathrm{CCN}$ proteins, can so dramatically alter the behavior of cancer cells, and if therapeutic peptides can be identified to modulate $\mathrm{CCN}$ function.

In the second Session on Tumor Biology, T. Shimo, (Dept of Oral and Maxillofacial Surgery, Okayama Graduate School of Medicine, Dentistry and Pharmaceutical Sciences), continued with new information on how CCN2 may contribute to osteolytic metastasis of breast cancer. Using the MDA-231 cell line and intracardiac injection to generate bone metastasis, it was shown that treatment with 
neutralizing anti-CCN2 markedly reduced the bone metastasis lesions, angiogenesis and osteoclast numbers therein. CCN2 was highly expressed in MDA-231 in bone lesions. PTHrP upregulated $\mathrm{CCN} 2$ expression. Application of pharmacological inhibitors of signaling pathways established that MAPK and PKC/PKA pathways were involved in the upregulation of CCN2 by PTHrP. Antibody to CCN2 reduced MDA-231 tumor growth by 30-50\% suggesting involvement of additional factors. To further add fuel to the fire, L. Desnoyers, (Genentech, San Francisco), then highlighted how CCN4 integrates activities with hyaluronan and CD44 to modulate breast cancer growth and metastasis. A mouse mammary tumor cell line model (4T1) was used and CCN4 is highly expressed in the stroma surrounding the cancer cells which are strongly positive for CD44 and HAS1/2 (hyaluronan synthase) suggesting a paracrine mechanism. CCN4 expressing 4T1 cells are highly migratory and invasive. CCN4 induced matrigel invasion of 4T1 which was inhibited by antibody to CCN4. Forced CCN4 expression in 4T1 upregulates CD44, and this correlates with increased growth, invasiveness and metastasis to lung and bone (other sites as well) from mammary site primaries. Thus, it would appear that aggressive breast $\mathrm{CA}$ exploits $\mathrm{CCN} 1$ and $\mathrm{CCN} 2$, and perhaps $\mathrm{CCN} 4$, for malignant progression, which may be counteracted by other $\mathrm{CCN}$ family members, eg, $\mathrm{CCN} 3$ and CCN5. Knowing how to manipulate $\mathrm{CCN}$ expression may be key to developing novel therapeutic approaches based on CCN structural biology. This Session then ended with a presentation by U. Kees, (Div of Children's Leukemia and Cancer Research, University of Western Australia, Perth), on CCN2 in pre-B ALL in children. This tumor can be generally successfully treated by chemotherapy. Microarray analysis of pre-B ALL compared with normal control CD34+/CD19+ cells identified CCN2 to be $~ 19-37$ fold higher expressed in pre-B ALL and as confirmed by qRTPCR. Comparison with other data sets confirmed the overexpression and correlation with expression of other subsets, MLL, TEL-AML, BCR-ABL. A large set of cell lines were examined where PER-377 was CCN2+ and Jurkat (T-ALL) was CCN2-. Western blots revealed a number of bands $30 \mathrm{kDa}, 37 \mathrm{kDa}$, etc suggesting potentially functional variants. CCN2 was secreted by ALL cells raising the question whether this could be a biomarker in ALL or responsible for distant physiological effects since CCN2 can promote a plethora of connective tissue responses. The ALL studies further support the functional significance of distinct $\mathrm{CCN}$ proteins in cancer.

In the first Session on Pathobiology, (Fibrosis), a subject area of long standing interest and intensive research activity in the CCN field, M. Mukoyama, (Dept of Medicine and Clinical Science, Kyoto University), started the talks off with a presentation on how $\mathrm{CCN} 1$ and $\mathrm{CCN} 2$ contribute to glomerular remodeling and renal disease. Glomerular fibrosis in kidney disease is a serious complication. First, it was shown that administering antisense $\mathrm{CCN} 2$ by hydrodynamic gene transfer, via the renal vein, effectively blocks CCN2 expression and thereby interstitial fibrotic changes, but not TGF $\beta 1$. Subtractive hybridization revealed that $\mathrm{CCN} 1$, as upregulated during anti-Thy-1 induced glomerulonephritis, is expressed specifically in podocytes in the early regeneration stages. CCN1 appears to inhibit migration of podocytes and induces their differentiation. A CCN1 transgenic mouse showed less nephropathy under challenge. In contrast, in diabetic nephropathy $\mathrm{CCN} 1$ is downregulated. This data points to a dual role of $\mathrm{CCN} 1$ and $\mathrm{CCN} 2$, i.e., renoprotection and disease progression, perhaps dependent upon accompanying inflammatory conditions. Again, it may be critical to assess other $\mathrm{CCN}$ proteins, like $\mathrm{CCN} 3$, which are expressed in the glomerulus to get a fuller picture of how the CCN homeostasis is disturbed. I. Klaassen, (Dept of Ophthalmology, University of Amsterdam), then spoke on how treatment of diabetic retinas, in a streptozocin induced diabetic rat model, with aminoguanidine, modulated expression of CCN family of proteins. Cytotoxic readout of streptozocin by measurement of advanced glycation products (AGE) indicated that $\mathrm{CCN} 1$ and $\mathrm{CCN} 2$ were downstream effectors of AGEs. Interestingly, 28, 22, and $11 \mathrm{kDa}$ variants of CCN1 were identified in tissues as well as in cultured microvascular cells. CCN2 is expressed in pericytes and microglia, and diabetic CCN2+/- mice develop reduced basement membrane thickening. Basement thickening seen at the preclinical stage suggests, as in other models, effects of $\mathrm{CCN}$ proteins on ECM structure and likely function. P-S Ng (Nosan Corp) using a mouse model of exogenous TGF- $\beta$ induced systemic sclerosis (skin fibrosis) demonstrated that neutralizing antibody to native CCN2 (produced by DNA immunization), especially those recognizing modules 1 (IGFBP) and 2 (VWC), could dramatically ameliorate the fibrotic response. Since most circulating $\mathrm{CCN} 2$ is carried by platelets, this is an accessible pool. Such reagents may be effective in other experimental models and potentially amenable to clinical application. H. Okada, (Dept of Nephrology, Saitama Medical School), reemphasized the fibrotic role of CCN2 in progressive kidney diseases and showed that tubular epithelium also expresses CCN2. A TGF $\beta$ responsive, cisregulatory element active in tubular cells was identified in the cen 2 promoter, in addition to BCE-1 and SBE sites. The message here is that $\mathrm{CCN} 2$ expression and degree of expression may be differentially regulated in different kidney cell types dependent upon stoichiometry of the regulatory factors, thus modulating the severity of the fibrotic changes. If this can be proven then treatments would require careful titration of therapeutics. 
In the last Session of the meeting, the second one on Pathobiology (Fibrosis), H. Yokoi, (Dept of Medicine and Clinical Science, Kyoto University), continuing along the kidney pathobiology theme, showed evidence that overexpression of CCN2 in podocytes leads to deterioration of glomerular function in a mouse model of diabetic nephropathy where CCN2 transcription was placed under control of the nephrin promoter. These mice were then subjected to streptozocin induced nephropathy and by 12 week had developed severe albuminuria. Histological analysis showed increased mesangial cell expansion and reduced numbers of podocytes as well as vacuolization of podocytes, a likely indicator of cell damage that could lead to cell death. Disarranged podocin indicated compromised filtering functions. The findings suggest that CCN2 overexpression accelerated diabetic changes and loss of glomerular function. M.S. Ahmed, (Institute for Surgical Research, Rikshospitalet University Hospital, University of Oslo), then brought attention to the serious problem of myocardial fibrosis where CCN2 also plays a role. In the CCN2 transgenic mouse model heart weight is lower and major functional defects are seen in the left ventricle coinciding with fibrotic changes and increased microvascularization. Affymetrix array analysis revealed 170 genes differentially regulated and one, GDF15 (TGF $\beta$ family), was significantly upregulated. Downregulation of EGF and upregulation of HIF $1 \alpha$ and GRK5, TIR4, and pSMAD-2 could explain pathological changes to the cardiac tissue. In a model of pressure overload using abdominal aorta banding, heart weight was also reduced in the CCN2 transgenic mice while CCN1 expression was found to be elevated. Non-transgenic banded mice showed significantly increased left ventricular end-diastolic diameters but not the CCN2 transgenic group, suggesting protection by $\mathrm{CCN} 2$ during pressure overload. This alternate pattern of $\mathrm{CCN}$ expression and function occurs dependent upon the tissue insult is seen here again raises the possibility that $\mathrm{CCN}$ proteins can cooperate or antagonize in multiple ways. Thus it may be necessary to run $\mathrm{CCN}$ expression profiles routinely to get a better insight into the physiological and pathological functions of $\mathrm{CCN}$ proteins. M. Arai, (Dept of Medicine and Biological Science, Gunma University Graduate School of Medicine), ended the Session and platform presentations in the CCN Workshop by speaking on role of CCN2 in development of cardiac fibrosis, and importantly, how CCN2 secretion could be used as a diagnostic and potentially prognostic indicator of ongoing fibrosis. In a rat pressure-overload cardiac hypertrophy model, CCN2 expression was compared to BNP (an anti-fibrotic peptide). The higher $\mathrm{CCN} 2 / \mathrm{BNP}$ ratios correlated with left ventricle stiffness and myocardial fibrosis. CCN2 secreted by cardiomyocytes could induce collagen synthesis by cardiac fibroblasts providing a mechanism for the observed fibrosis. In a series of 49 patients $\mathrm{CCN} 2$ protein levels in blood plasma, measured by ELISA, correlated positively with heart failure. These observations suggest that plasma CCN2 may serve as a clinical marker of fibrotic changes that compromise heart function or indicate heart failure.

The Poster Session offered participants the opportunity to present other $\mathrm{CCN}$ studies or to expand upon their work with additional data. New information is presented here. $\mathrm{M}$. Blea from Castellot's group in Boston showed results on efforts to generate a functional CCN5 knockdown in a smooth muscle tissue specific context since hyperproliferation of smooth muscle cells is inhibited by CCN5. M. Gray from the same Boston laboratory showed results on comparing expression of $\mathrm{CCN} 5$ and $\mathrm{CCN} 2$ in rodent tissues. Interestingly, there was extensive overlap in expression in multiple cell types suggesting that, if these CCN proteins did act differentially, they could operate through different mechanisms and could also have additional and specific biological functions. Nuclear/perinuclear localization of CCN5 and CCN2 was also observed, suggesting again their possible nuclear function. J. Malmquist from the same group provided other provocative information showing significant embryonic expression of CCN5, which was somewhat unexpected considering the antiproliferative activity of CCN5 in vitro on smooth muscle cells. However, it would appear that CCN5 alone is not sufficient to prevent cell proliferation and thus these observations raise the interesting question of $\mathrm{CCN}$ protein interactions and physiologically relevant concentrations. H. Kawaki from Takigawa's group presented results from studies of $\mathrm{CCN}$ proteins in the chondrogenic zones of $\mathrm{CCN} 2$ mutant mice. This was coupled with studies on isolated chondrocytes and overall the evidence points to differential expression with $\mathrm{CCN} 3$ strongly upregulated and slight $\mathrm{CCN} 6$ increase in $\mathrm{CCN} 2$ deleted cells suggesting that there are possible collaborations of $\mathrm{CCN}$ proteins in effecting terminal differentiation. K. Kawata from the same Okayama group showed that the recently identified receptor for CCN2, namely LRP1, appears in the HCS-2/ 8 cell line to internalize $\mathrm{CCN} 2$ where it is re-localized through membrane compartments and into the nucleus. Treatment of HCS-2/8 with siRNA for LRP1 led to decreased expression of aggrecan and col2al suggesting a possible transcriptional regulatory effect of LRP1, which also speaks to the issue of whether $\mathrm{CCN}$ proteins themselves can modulate transcription. N. Lazar from Prof. Perbal's laboratory addressed a critical issue in the $\mathrm{CCN}$ field, that of the availability of antibodies with high specificity and experimental utility. Lazar produced affinity purified polyclonal antibodies to each domain of $\mathrm{CCN} 3$ and showed clear biochemical evidence of their specificity and the 
expression and localization of CCN3 and its variants in transfected cells. These reagents should prove invaluable for further functional studies and to address the cellular roles for amino truncated forms as well as protein interactions. M. Ono and colleagues, (Okayama), examined the response of human bone marrow stromal cells (hBMSC) to substratum adsorbed $\mathrm{CCN} 2$ in vitro and in hydroxyapatite suspendend hBMSC as xenografts in nude mice. Adhesion of hBMSC to CCN2 activated p38MAPK and thereby migration and survival in vitro and in vivo suggesting a role for CCN2 in facilitating hBMSC mediated bone regeneration. Also, T. Fujisawa from Okayama presented in vitro and in vivo results describing the utility of $\mathrm{CCN} 2$ in auricular cartilage reconstruction, suggesting wider utility of CCN2 in mesenchymal tissue regeneration. Such effect is also supported by the findings presented in $\mathrm{T}$. Hattori's poster presented by E. Aoyama from Okayama. The integrin-CCN2 connection proposed by L. Lau seems to be working in chondrocyte adhesion events as well, in collaboration with fibronectin through its CT module interface. J. Russo and J. Castellot, (Boston) used a $21 \mathrm{~K}$ gene microarray analysis and qRT-PCR to identify and validate potential downstream effectors of the CCN5 inhibitory effect on smooth muscle growth, in particular cells contributing to the formation of leiomyomas (fibroids). Although not yet confirmed it would appear that CCN5 induces expression of multiple extracellular matrix genes as well as MMPs and regulatory genes corresponding to the anti-proliferative and anti-migratory effects of CCN5. T. Yanagita from Dr. Takigawa's group reported on the identification of alternative mRNAs of CCN4 representing variants lacking $\mathrm{VWC}$ or $\mathrm{VWC} / \mathrm{TSP} /$ part of the IGFBP module, suggesting that alternative forms of $\mathrm{CCN} 4$ may participate in chondrocyte differentiation, endochondral ossification, and possibly malignant transformation, although it remains to be determined how such variants can modify chondrocyte functions and induce transformation. Once again, the finding of short forms of $\mathrm{CCN}$ proteins with potentially additional functions is intriguing and is becoming more of a common theme in the field.

Summary Synopsis: It is becoming increasingly evident that there are complex patterns of $\mathrm{CCN}$ family expressions correlating with diverse biological functions in multiple tissue types. Furthermore, different $\mathrm{CCN}$ proteins may be either cooperating to effect specific biological behaviors or antagonizing each other to tightly regulate cell reactions that lead to pathological processes. Although CCN2 figures prominently in induction of the fibrotic process it may also conversely inhibit this process in certain cell and tissue injury contexts. This points to the importance of identifying protein partners that ultimately determine the functional contribution of any $\mathrm{CCN}$ protein. Expanding work on $\mathrm{CCN} 3, \mathrm{CCN} 4$, and CCN5 is establishing new avenues of functional involvement of $\mathrm{CCN}$ family and illustrating that $\mathrm{CCN} 3$ and CCN5 may be counteracting the activities of the other $\mathrm{CCN}$ members. Significantly, evidence was presented that isoforms or variants of $\mathrm{CCN}$ proteins may be playing additional critical roles in normal and malignant cells and directly effecting transcriptional changes. The $\mathrm{CCN}$ field is now realizing the benefits of standardizing reagents and especially development of domain specific antibodies, although much more has to be done in this area. Finally, from a clinical perspective, there is growing appreciation of the potential utility of $\mathrm{CCN}$ proteins as biomarkers of pathological processes, as deliverable therapeutics, and as lead macromolecules for identifying new pathways and factors mediating pathological processes. Thus the Fourth International Workshop ended on a high note as the participants anticipate continuing progress in our knowledge of CCN biology. Future workshops already scheduled for Toronto, Belfast and Sydney will hopefully spark increased interest from investigators outside the $\mathrm{CCN}$ field who can now appreciate the broad involvement of the $\mathrm{CCN}$ family of genes in diverse normal and pathological processes. 